\title{
Diagnosis and treatment of posttraumatic epilepsy
}

Khaydarova Dildora Kadirovna ${ }^{1}$, Khodjieva Dilbar Tadjiyevna ${ }^{1}$

${ }^{1}$ Department of Neurology and psychiatry, Bukhara Medical Institute, Republic of Uzbekistan.

\section{Khaydarov Nodirjon Kadirovich ${ }^{2}$}

${ }^{2}$ Tashkent Institute of Advanced Medical Education, Republic of Uzbekistan.

\section{Email address:}

dildora_doktor@mail.ru (Khaydarova Dildora Kadirovna)

\section{To cite this article:}

Khaydarova Dildora Kadirovna, Khodjieva Dilbar Tadjiyevna, Khaydarov Nodirjon Kadirovich. Diagnosis and treatment of posttraumatic epilepsy. Journal of research in health science. Vol. 1, No. 2, 2018, pp. 45-51. DOI 10.26739/2523-1243

\section{doi hittp://dx.doi.org/10.26739/2523-1243/-2017-1-2-7}

\begin{abstract}
Both neurologists and psychiatrists often diagnose posttraumatic epilepsy (PTE). This is due to the high rate of craniocerebral trauma (CCT), especially mild; in a patient with a first epileptic seizure, the presence of a history of CCT often leads to an incorrect diagnosis of PTE and inadequate treatment. The article presents our own data and literature review concerning the diagnosis and treatment of PTE.
\end{abstract}

Keywords: posttraumatic epilepsy, craniocerebral trauma, amnesia, myoclonus

\section{Introduction}

Posttraumatic epileptic seizures are divided into early seizures that appear in the first 7 days after CCT, and late, developing 1 week or more after trauma. This division is clinically justified, since the risk of recurrence of early seizures is low, whereas in $80 \%$ of patients who undergone epileptic paroxysm after 1 week after CCT, PTE is formed in the next 2 years [1].

PTE is a chronic disease characterized by repeated unprovoked attacks of motor, sensitive, vegetative and mental functions that result from excessive neuronal discharges. The share of PTE accounts for $20 \%$ of all symptomatic forms of epilepsy. It is most common among patients aged 15-24 years. The probability of PTE development varies from 1.9 to $37 \%$ and depends on the severity and nature of the CCT $[2,3]$. The frequency of development of PTE for 30 years with a slight trauma (loss of consciousness to 30 minutes) is $2.1 \%$, with moderate (loss of consciousness from 30 minutes to 24 
Khaydarova Dildora Kadirovna, Khodjieva Dilbar Tadjiyevna, Khaydarov Nodirjon Kadirovich. Diagnosis and treatment of posttraumatic epilepsy.

hours, or damage to the skull) $-4.2 \%$, with severe (loss of consciousness, or post-traumatic amnesia> $24 \mathrm{~h}$, or intracranial hematoma, or verified brain contusion) - $16.7 \%$ [4]. Heavy CCT increases the risk of epilepsy 29 times compared with general population, while the lungs - in 1,5 times [5]. PTE in $40 \%$ of cases debuts in the first 6 months after injury, $50 \%$ in the first year and $80 \%$ in the first 2 years [6]. However, the period of increased risk of PTE development is much longer and depends on the severity of CCT: after a minor trauma it is 5 to 10 years, after an average injury - about 10 years, and after severe - up to 20 years [4].

\section{Diagnostics}

In the management of a patient who has a history of CCT and suffered a first attack of PTE, it is necessary to solve three main tasks: 1) to establish whether this is an epileptic seizure; 2) exclude his nontraumatic etiology; 3) appoint an effective treatment.

\section{If PTE is supposed, then:}

1) confirm the diagnosis of epilepsy;

2) adjust the therapy (if it is prescribed);

3) consider the possibility of surgical treatment of epilepsy with pharmacoresistance.

\section{Clinical data}

With the development of a patient who has had an IBT, any paroxysm in the first place it is necessary to establish whether he was epileptic. Data on the nature of the attack is important to get both from the patient himself and from eyewitnesses.

Moreover, it is better that the eyewitness did not try to explain, but showed the motor manifestations that he observed in the patient. This technique helps to avoid mistakes.
It is necessary to ask the patient about the first signs of a paroxysmal event (the presence of an aura), its further development and post-seizure state (speech disorders, Todd's paresis, sleep). In some cases, before the development of the secondary generalized attack, the patient could have isolated auras, which he did not attribute to epilepsy and did not complain about them.

The presence of focal seizures before CCT calls into question the diagnosis of PTE. Myoclonus, especially those in the morning, are also uncharacteristic of PTE. In their presence, the generalized tonicclonic seizure that occurs after CCT is most likely not associated with trauma (especially mild), but rather indicates a diagnosis of juvenile myoclonic epilepsy (Yants syndrome).

In patients with severe CCT, severe cognitive disorders are often found in the form of memory impairment and speech functions (dysarthria, aphasia).

In the first case, patients forget about attacks, in the second they cannot talk about them. In patients with depressed consciousness (coma, vegetative status, and various types of mutism) it is difficult to identify and qualify paroxysmal events, and in accordance with the international classification of seizures at the time of the paroxysmal event, the patient must be conscious. Therefore, in such situations this classification is unacceptable and acquire special significance a detailed collection of data on the ictal event from eyewitnesses, as well as long-term monitoring of the videoelectroencephalogram (EEG) to confirm the epileptic nature of the paroxysms.

If the patient has seizures for a long time, it is necessary to answer the question whether paroxysmal events are epileptic. 
It is known that in patients undergoing CCT, the frequency of pseudo-epileptic (psychogenic) paroxysms is high. In one study, the frequency of psychogenic seizures in patients diagnosed with "pharmacoresistant PTE" reached 30\% [7]. There is evidence that the risk of developing psychogenic attacks is lightweight head injury [8], while the likelihood of epilepsy increases with increasing severity of injury. Differential diagnostics are helped by a detailed description of septic seizures: typical automatisms (oral, gestural), loss of consciousness, speech disorders, stereotyped nature of events. Extremely desirable is a video recording of paroxysm, which can be made by relatives of the patient (for example, on the video camera of the phone). An important distinctive feature of epileptic seizures is a stared look and wide-eyed eyes (staring), whereas in psychogenic paroxysmal events, patients often close their eyes. It is necessary to pay attention to the circumstances under which seizures occur, attachment to the sleep-wake cycle, provoking factors. However, it is only possible to confirm or exclude the diagnosis of epilepsy only based on data from video EEG monitoring with the recording of paroxysms. One patient may have a combination of epileptic and psychogenic seizures, which, according to the literature, is noted in 15-30\% of patients with psychogenic paroxysms [9]. At the same time, in most cases it is possible clearly distinguish between these two types of paroxysms [10]. A detailed analysis of septic seizures will suggest localization of the epileptic focus and confirm the clinical data with the results of neuroimaging methods of investigation - X-ray computer (CT) and magnetic resonance
(MRI) tomography. The coincidence of the zone of posttraumatic morphological lesions of the brain according to $\mathrm{CT}$ or MRI and a clinical topical diagnosis confirms the diagnosis of epilepsy.

Similar differential diagnosis is also performed with such paroxysmal states of non-epileptic genesis as special sleep disorders (parasomnia), paroxysmal dyskinesia, syncope.

\section{EEG}

Next in importance after the clinical data in the diagnosis of epilepsy are the results of EEG. Epileptic activity can be detected during the Interictal period (Interictal activity) and during an attack (ictal activity). In patients after CCT in the interictal period, various diffuse and local disturbances are revealed: slowing and disorganization of the background rhythm, regional periodic or prolonged deceleration. However, registration of interictalepileptiform patterns (IEP), which are highly specific for this disease, is especially important for the confirmation of epilepsy. There is noambiguous definition of IEP, but the following criteria are widely used $[11,12]$ :

1) Paroxysmal character (clearly distinguished from the background rhythm);

2) A sharp change in the polarity within a few milliseconds, which gives the IEP an "acute" form;

3) Duration, usually not exceeding $200 \mathrm{~ms}$ for sharp waves and $70 \mathrm{~ms}$ for spikes;

4) Recording from more than one electrode and the presence of an amplitude gradient on the scalp, which indicates the physiological nature of the electric field;

5) Negative polarity in the overwhelming majority of cases. 


\section{Khaydarova Dildora Kadirovna, Khodjieva Dilbar Tadjiyevna, Khaydarov Nodirjon Kadirovich.}

Diagnosis and treatment of posttraumatic epilepsy.

EEG data should always be interpreted in the context of a specific clinical situation. Thus, the presence of a bone defect leads to a sharp increase in the amplitude and sharpened character of physiological rhythms in the zone of craniotomy ("breach rhythm"), cysts and extensive foci of brain contusion in some cases create additional obstacles when registering the IEP and a false impression of lateralization of the zone of irrational changes.

In addition, some normal physiological patterns (lambda waves, benign epileptiform sleep phenomena, wicketspikes, "6 $\mathrm{Hz}$ phantom spikes", etc.) can satisfy the given IEP criteria, however, having a specific morphology, they are easily differentiated by the experienced an electroencephalographist.

IEP are very diverse. Common is a sharp change in rhythm that precedes the clinical manifestations of an epileptic attack or coincides with them. In some cases, subclinical patterns are recorded, i.e., not having obvious clinical manifestations. The recording of an attack in most cases is possible only with longterm registration using video EEG monitoring.

Probability of registration of IEP in patients without epilepsy (specificity of the method)

In adults without epilepsy, IEPs are very rarely recorded in $0.2-0.5 \%$ of cases, in children more often (2.2-3.5\%) [11]. The presence of focal lesions of the brain, in particular, the focus of injury after CCT, increases the probability of registration of IEP [13].

For children, centrally temporal adhesions, a photoparoxismal response, and generalized discharges are characteristic. These genetically determined phenomena are registered in $4 \%$ of healthy children aged 4 to 12 years $[14,15]$.

IEP is sometimes observed in patients taking high doses of chlorpromazine, lithium, clozapine, as well as with the abrupt withdrawal of barbiturates and benzodiazepines [12].

\section{Methods for increasing the sensitivity} of the EEG

To improve the registration of IEP with EEG, various "activating" (provoking) methods are used: hyperventilation, photostimulation and sleep deprivation, installation of additional electrodes, as well as recording in sleep and many hours (sometimes, many-days) EEG monitoring.

Hyperventilation is used in the absence of epilepsy; it allows identifying generalized "peek-wave" patterns in 50$80 \%$ of children [18]. The probability of recording focal IEPs (in particular, with PTE) hyperventilation increases by less than $10 \%$.

Focal occipital and idiopathic forms of epilepsy are sensitive to photo stimulation [19]. An important activating technique is a 24-hour sleep deprivation, which significantly increases the likelihood of IEP registration, not significantly increasing the risk of an attack. Against the background of deprivation of sleep, the stimulating effect of photo stimulation and hyperventilation is potentiated $[20,21]$.

The installation of additional electrodes (zygomatous, sphenoidal) is justified if the standard 10-20 system does not allow the registration of IEP, and is most commonly used for medial temporal epilepsy [22].

The probability of recording IEP increases with the increase in the duration of recording, for this purpose, long-term (sometimes, many-days) 
EEG monitoring is used [23]. When monitoring EEG recording in a dream. The importance of recording EEG sleep in patients with epilepsy has already been shown in the first works of E.L. Gibbs [24]. Now it is known that in $40 \%$ of patients with epilepsy without IEP during wakefulness they are recorded in a dream. There is no unequivocal opinion about which phase of sleep (superficial or deep) is more sensitive to epileptiform discharges. However, all authors agree that in the stage of rapid sleep the probability of the appearance of epileptiform activity is minimal, but it is more local in nature [20].

EEG monitoring is important not only in the diagnosis of epilepsy, but also in deciding whether to cancel the drug. It was shown that the detection of epileptiform activity in EEG monitoring in a patient with pharmacological remission indicates a high risk of relapse of epilepsy.

\section{Treatment}

Anticonvulsant therapy in patients with $\mathrm{BCC}$ is performed only after the diagnosis of PTE. The effectiveness of prophylactic use of antiepileptic drugs (AEP) has not been proven to date [25-27]. The only attack after CCT cannot be regarded as epilepsy, but the likelihood of a recurrence is high (up to $80 \%$ ). The decision to initiate drug therapy is taken with the patient; the risk of recurrence of the attack is discussed, on the one hand, and the possibility of side effects of anticonvulsant therapy, on the other. It should be remembered that the speed of decisionmaking on the beginning of antiepileptic treatment does not affect the possibility of achieving remission in the future. When choosing drugs preference is given to the most effective and safe. For the focal forms of epilepsy, it is carbamazepine (tegretol, finlepsin), oxcarbazepine (trileptal), and lamotrigine (lamitale, lamolept, lamitore). In a small part of patients with the phenomenon of secondary bilateral synchronization on EEG, valproates (depakin, convulex) are the best choice. Second-line drugs include topiramate (topamax, thorale), levithiracetam (keppra) and clonazepam; the last two are effective in myoclonic seizures, which, however, are rarely noted in PTE. In connection with frequent and pronounced side effects, barbiturates are preparations of the third stage [28].

PTE therapy is long, at least 2 years in children and 3-5 years in adults after the last attack, but in patients with a single paroxysm, this period may be shortened.

Begin treatment with a minimum therapeutic dose, with insufficient effectiveness, it is gradually increased under the control of the concentration of the drug in the blood (in Russia, the concentration of valproate, carbamazepine and barbiturates is available) to the maximum tolerated. If two courses of monotherapy are ineffective, they start treatment with two AEDs. However, the correct diagnosis and the appointment of an adequate treatment are only the first stage of PTE therapy. The problem of medicine, especially epileptology, where long regular medication is required, is noncompliance with medical prescriptions. According to data of different authors [29, 30], up to $40 \%$ of patients do not take drugs at the prescribed dose, skip their intake or refuse therapy altogether. Therefore, the priority task is the patient's motivation for regular AEP reception, as well as for observing the sleep regimen, avoiding the triggers of an attack 
Khaydarova Dildora Kadirovna, Khodjieva Dilbar Tadjiyevna, Khaydarov Nodirjon Kadirovich. Diagnosis and treatment of posttraumatic epilepsy.

of factors (for example, flashing lights at discotheques, etc.). To increase the adherence of patients to treatment, reduce the frequency of taking medications (two times better than three times), and develop a convenient reception stereotype, tied to any regular activity (food, walks, TV shows, etc.).

\section{References}

1. Haltiner A.M., Temkin N.R., Dikmen S.S.Risk of seizure recurrence after the first lateposttraumatic seizure. Arch Phys Med Rehabil1997;(78):835-40.

2. Zaitsev OS, Potapov AA, Shaginyan G. et al. Epileptic syndrome in patients with gunshot wounds. VoproNeurochir 2000; (2): 21-5.

3. Englander J., Bushnik T., Duong T.T. et al.Analyzing risk factors for late posttraumaticseizures: a prospective, multicenter investigation.Arch Phys Med Rehabil2003;(84):365-73.

4. Annegers J.F., Hauser W.A., Coan S.P. et al.A population-based study of seizures after traumaticbrain injuries. N Engl J Med1998;(38):20-4.

5. Herman S.T. Epilepsy after brain insult: targetingepileptogenesis. Neurology2002;59(Suppl. 5):21-6.

6. Beghi. E. Overview of Studies to PreventPosttraumatic Epilepsy. Epilepsia2003;44(Suppl. 10):21-6.

7. Hudak A.M., Trivedi K., Harper C. et al.Evaluation of seizure-like episodes in survivorsof moderate and severe traumatic brain injury. JHead Trauma Rehabil2004;(19):290-5.

8. Westbrook L.E., Devinsky O. Nonepilepticseizures after head injury. Epilepsia1998;(9):978-82.

9. Rotge J.Y., Lambrecq V., Marchal C. et al.Conversion disorder and coexisting nonepilepticseizures in patients with refractory seizures.Epilepsy Behav2009;(2):350P2.

10. Devinsky O., Sanchez-Villasenor F.,Vazquez B. et al. Clinical profile of patients withepileptic and nonepileptic seizures. Neurology1996;(6):1530-3.

11. Bao F.S., Gao J.M., Hu J. et al. ConfProcIEEE Eng Med Biol Soc. Automated epilepsydiagnosis using interictal scalp EEG2009;2009:6603-7.

12.Engel J.Epilepsy A ComprehensiveTextbook.Philadelphia: Lippincott Williams \&Wilkins, 2008.

13. Ronne-Engstrom E., Winkler T.ContinuousEEG monitoring in patients with traumaticbrain injury reveals a high incidence of epileptiformactivity. ActaNeurolScand2006;(1):47-53.

14. Okubo Y., Matsuura M., Asai T. et al.Epileptiform EEG discharges in healthy children:prevalence, emotional and behavioral correlates, and genetic influences. Epilepsia1994;(4):832-41.

15. Bali B., Kull L.L., Strug L.J. et al.Autosomal dominant inheritance of centrotemporalsharp waves in rolandic epilepsy families.Epilepsia 2007;48(12):2266-72.

16.Salinsky M., Kanter R., Dasheiff R.M.Effectiveness of multiple EEGs in supportingthe diagnosis of epilepsy: an operational curve.Epilepsia 1987;(4):331-4.

17. KozlovaAB, Arkhi povaNA, StepanenkoA.Yu.Preoperational neurophysiological examination of patients with epilepsy caused by damage to the frontal lobe. VoproNeurochir 2006; (1): 26-32.

18. Sadleir L.G., Scheffer I.E., Smith S. et al.EEG features of absence seizures in idiopathicgeneralized epilepsy: impact of syndrome, age,and state. Epilepsia2009;(6):1572P8.

19. Jayakar P., Chiappa K.H. Clinical correlationsof photoparoxysmal responses.ElectroencephalogrClinNeurophys1990;75:251-4.

20. Degen R., Rodin E.A. (eds.). Epilepsy, sleepand sleep deprivation. Epilepsy Res

1991;(Suppl. 2):1-280.

21. Ayvazyan SO, Shiryaev LS, Golovteev AL and others. Non-invasive video-EEG monitoring in the diagnosis of epilepsy in children. VoproNeurochir 2009; (1): 33-41.

22. Sperling M.R., Mendius J.R., Engel J.Mesial temporal spikes: a simultaneous comparisonof sphenoidal, nasopharyngeal, and earelectrodes. Epilepsia 1986;27:81-6.

23. Velis D., Plouin P., Gotman J. et al.Recommendations regarding the requirementsand applications for long-term recordings inepilepsy. Epilepsia2007;(2):379-84.

24. Gibbs E.L., Gibbs F.A. Diagnostic andlocalizing value of electroencephalographicstudies in sleep. Res PublAssoc Res NervMentDis 1947;(26):366-76. 
Journal of research in health science. 2018; 1 (2): 45-51.

25. Adelson P.D., Bratton S.L., Carney N.A. etal. Guidelines for the acute medical managementof severe traumatic brain injury in infants, children, and adolescents. The role of antiseizureprophylaxis following severe pediatrictraumatic brain injury. American Association forSurgery of Trauma; Child Neurology Society;International Society for PediatricNeurosurgery; International Trauma Anesthesiaand Critical Care Society; Society of CriticalCare Medicine; World Federation of PediatricIntensive and Critical Care Societies. PediatrCrit Care Med 2003;4(3 Suppl.):72-5.

26. Bratton S.L., Chestnut R.M., Ghajar J. etal. Guidelines for the management of severetraumatic brain injury. XIII. Antiseizure prophylaxis.Brain Trauma Foundation; AmericanAssociation of Neurological Surgeons; Congressof Neurological Surgeons; Joint Section onNeurotrauma and Critical Care, AANS/ CNS. JNeurotrauma2007;(24 Suppl. 1):83-6.

27. Warden D.L., Gordon B., McAllister T.W. etal. Guidelines for the pharmacologic treatmentof neurobehavioral sequelae of traumatic braininjury. Neurobehavioral Guidelines WorkingGroup $\mathbf{J}$ Neurotrauma 2006;23(10):1468-501.

28. Zaitsev OS, Psychopathology of severe craniocerebral injury. Diss. ... Doct. honey. sciences. M., $2011 ; 324 \mathrm{p}$.

29.Cramer J.A., Glassman M., Rienzi V.Therelationship between poor medication complianceand seizures. EpilepsyBehav2002;(4):338-42.

30. Kotov A.S. Compliance in patients with epilepsy. JourNeuro and Psychiatrist 2010; (11): 46-8. 